


\section{UNIVERSITY OF WASHINGTON \\ DEPARTMENT OF OCEANOGRAPHY \\ Seattle, Washington 98105}

Technical Reports

Nos. 162, 163, 164,

165,166 , and 167

A COMPILATION OF ARTICLES REPORTING RESEARCH SPONSORED

BY THE OFFICE OF NAVAL RESEARCH

Office of Naval Research

Contracts Nonr-477(10)

and Nonr-477(37)

Project NR 083012

Reference $\mathrm{M} 66-76$

December 1966

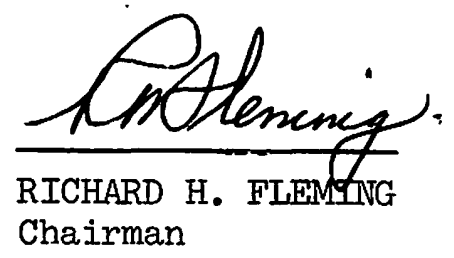

Reproduction in whole or in part is permitted for any purpose of the United States Government 
ARTICLES REPORTING RESEARCH SPONSORED BY THE OFFICE OF NAVAL RESEARCH

Technical Report No. 162

QUASIGEOSTROPHIC FREE OSCTLIATIONS IN ENCLOSED BASINS, by Maurice Rattray, Jr. and Robert I. Charnell. Journal of Marine Research, 24(1):82-103. 1966.

$$
\text { Technical Report No. } 163
$$

PALAGONITE TUFFS (HYALOCLASTITES) AND THE PRODUCTS OF POST-ERUPTIVE PROCESSES, by $Y$. Rammohanroy Nayudu. Bulletin Volcanologique, 27:391-410 +5 plates. 1964.

Technical Report No. 164

CARBONATE DEPOSITS ON PLANTAGENET BANK NEAR BERMUDA, by M. Grant Gross . Geological Society of America Bulletin, 76:1283-1290. 1965.

Technical Report No. 165

DIGGING CHARACTERISTICS AND SAMPLING EFFICIENCY OF THE $0.1 \mathrm{~m}^{2}$ VAN VEEN GRAB, by Ulf Lie and Mario M. Pamatmat. Limnology and Oceanography, 10 (3):379-384. 1965 .

Technical Report No. 166

GRAVITATIONAL CIRCULATION IN STRAIIS AND ESTUARIES, by Donald V. Hansen and Maurice Rattray, Jr. Journal of Marine Research, 23 (2):104-122. 1965.

Technical Report No. 167

OXYGEN AND CARBON ISOTOPIC COMPOSITION OF LIMESTONES AND DOLOMITES, BIKTNI AND ENTWETOK ATOLIS, by M. Grant Gross and Joshua I. Tracy, Jr. Science, ijl(3714): 1082-1084. 1966. 
UNIVERSITY OF WASHINGTON

DEPARTMENT OF OCEANOGRAPHY

TECHNICAL REPORT NO. 166

REPRINT FROM JOURNAL OF MARINE RESEARCH, VOLUME 23, 2, 1965 


\title{
Gravitational Circulation in Straits and Estuaries
}

\author{
Donald V. Hansen and Maurice Rattray, Jr. \\ Department of Oceanography \\ University of Washington \\ Seattle, Washington
}

\begin{abstract}
A coupled set of partial differential equations and associated boundary conditions is written to describe circulation and salt-flux processes for estuaries in which turbulent mixing results primarily from tidal currents. Similarity solutions, motivated by characteristic salinity distributions observed in estuaries, are obtained for this set of equations and are compared with observational data. The circulation is separated into modes analogous to the barotropic, baroclinic, and Ekman modes of oceanic circulation. The salinity distribution, although coupled to the velocity distribution, is found to vary independently of it as well. The theoretical results are discussed in regard to: (i) correlation between the vertical variations of mean velocity and salinity, (ii) the role of this correlation in maintaining the steady-state salinity distribution in estuaries, and (iii) some implications for computations of flushing and dispersion of contaminants.
\end{abstract}

I. Introduction. The longitudinal salinity distribution in many coastal-plain estuaries takes the general form of the hyperbolic tangent function, being almost linear with the maximum gradient in the central part, and tailing off gradually to terminal values in the river and ocean. In the central region the vertical salinity stratification is nearly independent of longitudinal position, while in the terminal regions it is proportional to the departure of the sectional mean salinities from their respective asymptotic values. An example of this characteristic salinity distribution with its three regimes is shown in Fig. 1 , which is based on Pritchard's (1954a) data from the Delaware River model.

The basic nontidal circulation associated with, and active in, maintaining the salinity distribution in estuaries consists of a seaward flow of river water

I. Contribution No. 349 from the Department of Oceanography, University of Washington. This research was supported in part by the National Science Foundation under grants GP-I 101 and GP-3549 and in part by the Office of Naval Research under Contracts Nonr-477(10) and NoNR-477(37)Project NR 083-012.

Accepted for publication and submitted to press 4 April 1965. 


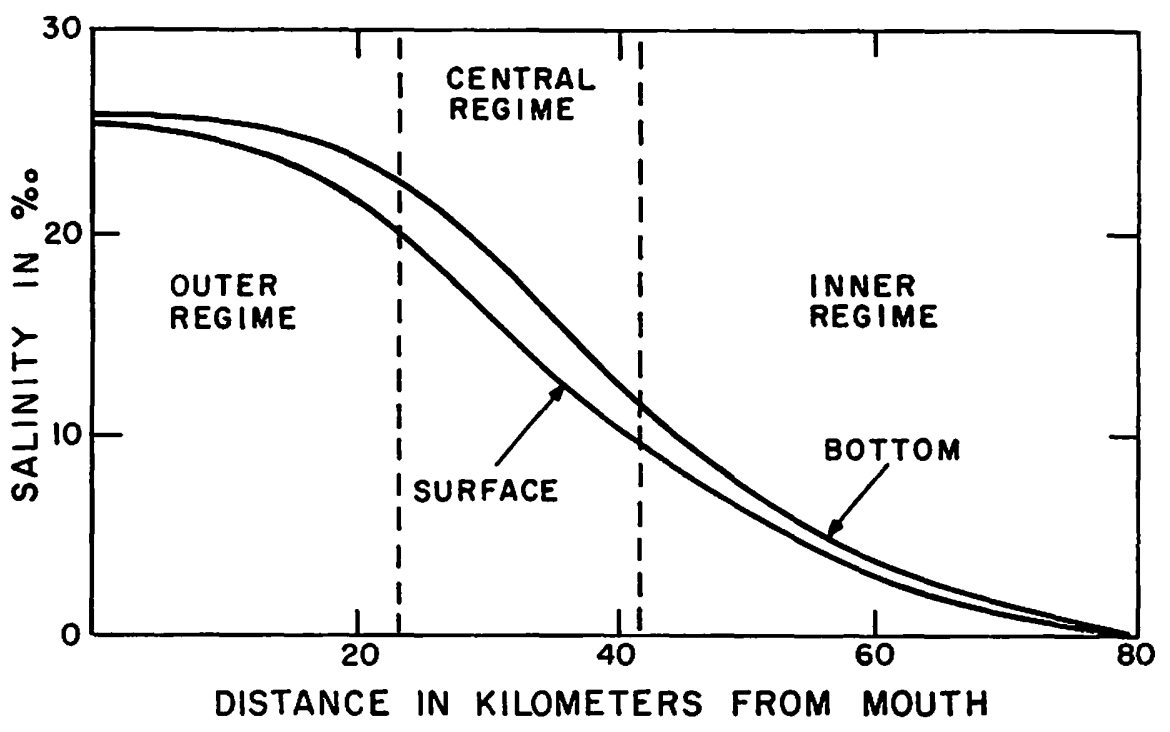

Figure 1. Longitudinal salinity distribution in the Delaware River (from Pritchard 1954a).

and a system of currents induced by the density difference between freshwater and seawater. Analysis of the estuarine regime therefore constitutes a problem of both forced and free convection. One-dimensional mathematical models, such as that of Arons and Stommel (1951) or Ippen and Harleman (196r), relate to integral properties of the forced flow and suppress or obscure the freeconvection aspect of the problem, i.e., the influence of density gradients on the velocity distribution; such models can therefore adequately portray conditions only in the innermost part of shallow estuaries, where such influence is slight. The effect of density gradients on the velocity distribution has been considered by O'Brien (1952) and Abbott (1960) without reference to the role of currents in maintaining the salinity distribution. Agnew (196I) developed separately two aspects of the free-convection problem: (i) the influence of an assumed density gradient on tidal currents in estuaries, and (ii) the tendency of an assumed free-convection velocity gradient and horizontal density gradient to induce vertical density stratification. The interdependence of the salinity and velocity fields was recognized in the dynamical equations employed by Rattray and Hansen (1962), who developed the free-convection aspect of the problem but suppressed the forced-convection aspect to obtain an approximate solution for the outer regime, in which it was again necessary to assume knowledge of the horizontal density gradient.

This paper presents some solutions of the basic differential equations for the central and inner regimes; both forced and free convections are considered, and two-dimentional salinity distributions are derived from external parameters. 
Some of the results apply equally well to sea straits and narrows having strong tidal currents and well-defined water and density budgets.

\section{Notation}

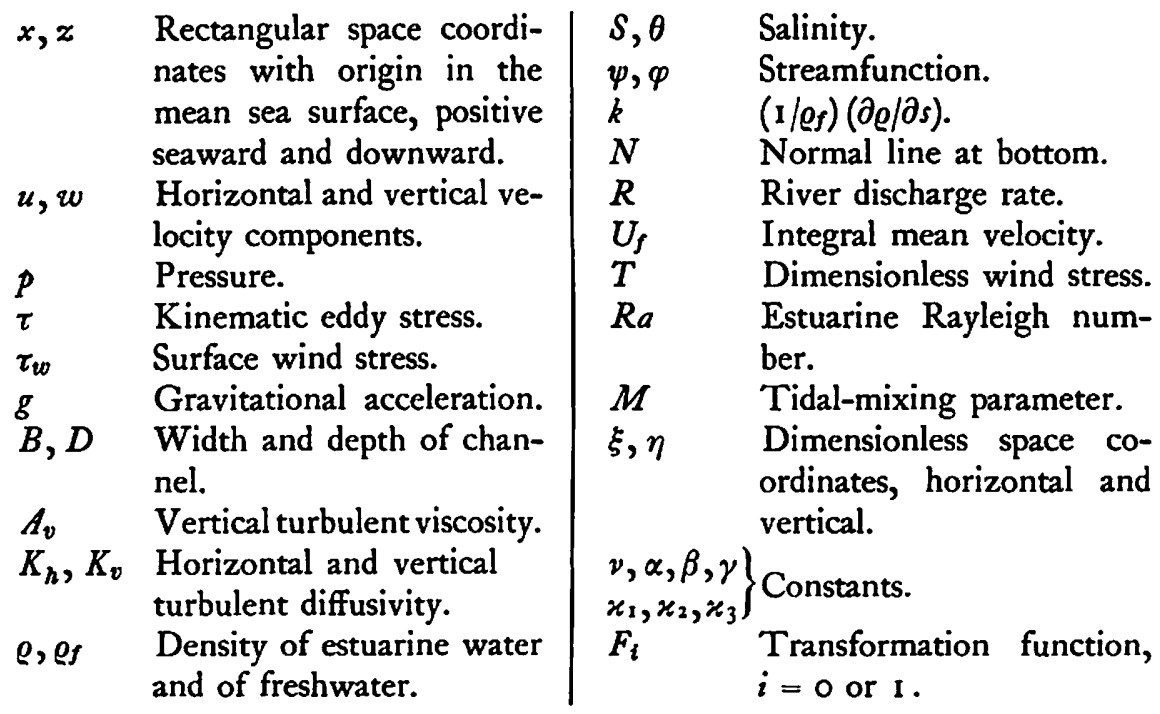

II. Formulation. Consideration is given to an idealized coastal-plain estuary that is rectilinear and is sufficiently narrow so that conditions are in effect laterally homogeneous. Tidal currents are assumed to be the predominant cause of turbulent mixing but to have no other influence upon the net circulation in the estuary. Turbulent exchanges are therefore expressed as products of the gradients of the mean value of the respective property over a tidal cycle and exchange coefficients that are independent of local properties of the mean flow; these conditions are most likely to be approached in regions of relatively uniform stratification.

Studies by Pritchard (1956) indicate that the dynamic balance of forces in a coastal-plain estuary can be expressed by

$$
\frac{1}{\varrho} \frac{\partial p}{\partial x}=\frac{\partial \tau}{\partial z}=\frac{\partial}{\partial z}\left(A_{v} \frac{\partial u}{\partial z}\right)
$$

and

$$
\frac{1}{\varrho} \frac{\partial p}{\partial z}=g \text {. }
$$

The equations for conservation of water and salt can be written:

$$
\frac{\partial}{\partial x}(B u)+\frac{\partial}{\partial z}(B w)=0,
$$


and

$$
B\left(u \frac{\partial s}{\partial x}+w \frac{\partial s}{\partial z}\right)=\frac{\partial}{\partial x}\left(B K_{h} \frac{\partial s}{\partial x}\right)+\frac{\partial}{\partial z}\left(B K_{v} \frac{\partial s}{\partial z}\right)
$$

the linear expression,

$$
\varrho=\varrho_{f}(\mathrm{I}+k S),
$$

is an adequate approximation to the equation of state for estuarine water.

Equation (3) is satisfied by introduction of the transport streamfunction, $\psi$, such that

$$
B w=\psi_{x} \text { and } B u=-\psi_{z},
$$

where subscripts are used to denote partial differentiation with respect to the subscript.

Eliminating $p$ and $\varrho$ among (1), (2), and (5), and using the Boussinesq approximation, we obtain the governing equations for the circulation and salt balance:

$$
\begin{gathered}
{\left[A_{v}\left(\frac{1}{B} \psi_{z}\right)_{z}\right]_{z z}+g k S_{x}=0,} \\
\psi_{x} S_{z}-\psi_{z} S_{x}=\left(B K_{h} S_{x}\right)_{x}+\left(B K_{v} S_{z}\right)_{z} .
\end{gathered}
$$

Boundary conditions to be satisfied are: no slip at the bottom, shearing stress equal to wind stress at the free surface, net transport equal to river flow, and zero normal salt flux at the boundaries. These conditions are expressed by:

$$
\left.\begin{array}{ll}
\text { at } z=D(x) ; & \psi=\psi_{N}=0, \\
\text { at } z=0 ; \psi=R, & A_{v}\left(\frac{\psi_{z}}{B}\right)_{z}=\tau_{w},
\end{array}\right\}
$$

and any two of

$$
\begin{aligned}
& \text { at } \quad z=D(x) ; \quad S_{N}=0, \\
& \text { at } \quad z=0 ; B K_{v} S_{z}-S \frac{d R}{d x}=0, \\
& \quad \int_{0}^{D(x)}\left(S \psi_{z}+B K_{h} S_{x}\right) d z=0 .
\end{aligned}
$$

We seek solutions that portray the transition from river to oceanic conditions, i.e., for $S_{x}>0$. Three types of similarity solutions having this property will be developed. These are separable solutions that require particular $x$ dependence of the river discharge, estuary width, and exchange coefficients. The particular conditions required, called similarity conditions, indicate rela- 
tions among the external parameters that may be expected to result in particular salinity and velocity distributions. The technique permits the width and the exchange coefficients to vary with depth; however, for mathematical simplicity in showing the basic relations, we shall consider only the idealized case of an estuary having a rectangular cross section and the exchange coefficients independent of depth.

IIIa. The Central Regime; Theory. It may be assumed without loss of significance that the width, depth, and river flow are all constant in the central regime. Solutions of equations (6) and (7) are sought in the form

$$
\left.\begin{array}{c}
\psi(x, z)=R \varphi(\eta), \\
S(x, z)=S_{o}[\nu \xi+\theta(\eta)],
\end{array}\right\}
$$

where $\nu$ is a constant, $\eta=z / D$ and $\xi=R x / B D K_{h o}$ are dimensionless coordinates, and zero subscripts indicate values at $x=0$.

The similarity conditions for distributions of this type are: the vertical exchange coefficients, $A_{v}$ and $K_{v}$, cannot vary along the estuary, but the horizontal exchange coefficient, $K_{h}$, must increase seaward at a rate equal to the integral mean velocity or freshwater discharge velocity, i.e.,

$$
\frac{d}{d x} K_{h}=\frac{R}{B D}=U_{f} .
$$

Seaward increase of turbulent diffusion in an estuary with a constant cross section may result from a corresponding increase in tidal velocity and excursion, but this result should not be confused with the increase in the apparent diffusion coefficients observed by Preddy (1954), Kent (1958), or Ippen and Harleman (196I).

The ordinary differential equations and boundary conditions obtained from (6), (7), (8), and (9) are:

$$
\begin{aligned}
& \left.\begin{array}{c}
\varphi^{\prime \prime \prime \prime}+\nu R a=0, \\
M \theta^{\prime \prime}+\nu\left(\varphi^{\prime}+1\right)=0,
\end{array}\right\} \\
& \varphi(1)=\varphi^{\prime}(1)=0, \varphi(0)=1, p^{\prime \prime}(0)=T,
\end{aligned}
$$

and any two of

$$
\left.\begin{array}{rl}
\theta^{\prime}(0) & =0, \\
\theta^{\prime}(\mathrm{I}) & =0, \\
\rho^{\prime} \theta d \eta & =0,
\end{array}\right\}
$$


where primes indicate differentiation with respect to $\eta$. These equations are characterized by three dimensionless parameters: $T=B D^{2} \tau_{w} / A_{v} R$ is the dimensionless wind stress, $R a=g k S_{0} D^{3} / A_{v} K_{h o}$ is an estuarine analog of the Rayleigh number, and $M=K_{v} K_{h 0} B^{2} / R^{2}$ denotes a ratio of tidal mixing to river flow.

It is convenient to choose the mean value of the time-average salinity over the section at $x=0$ for the reference salinity $S_{0}$ in (10). Solutions for the horizontal velocity and salinity distributions satisfying (12), (13), and ( 14 i, ii) then take the forms:

and

$$
\frac{u}{U_{f}}=-\frac{d \varphi}{d \eta}
$$

$$
\frac{S}{S_{0}}=1+\nu \xi+\frac{\nu}{M}\left[\left(\eta-\frac{1}{2}\right)-\frac{1}{2}\left(\eta^{2}-\frac{1}{3}\right)-\int_{0}^{\eta} \varphi d \eta+\int_{0}^{1} \int_{0}^{\eta} \varphi d \eta^{\prime} d \eta\right]
$$

where

$$
\varphi(\eta)=\frac{1}{2}\left(2-3 \eta+\eta^{3}\right)-\frac{T}{4}\left(\eta-2 \eta^{2}+\eta^{3}\right)-\frac{\nu R a}{4^{8}}\left(\eta-3 \eta^{3}+2 \eta^{4}\right) .
$$

The longitudinal top and bottom salinity distribution given by (16) for the case of no wind stress is shown in Fig. 2.

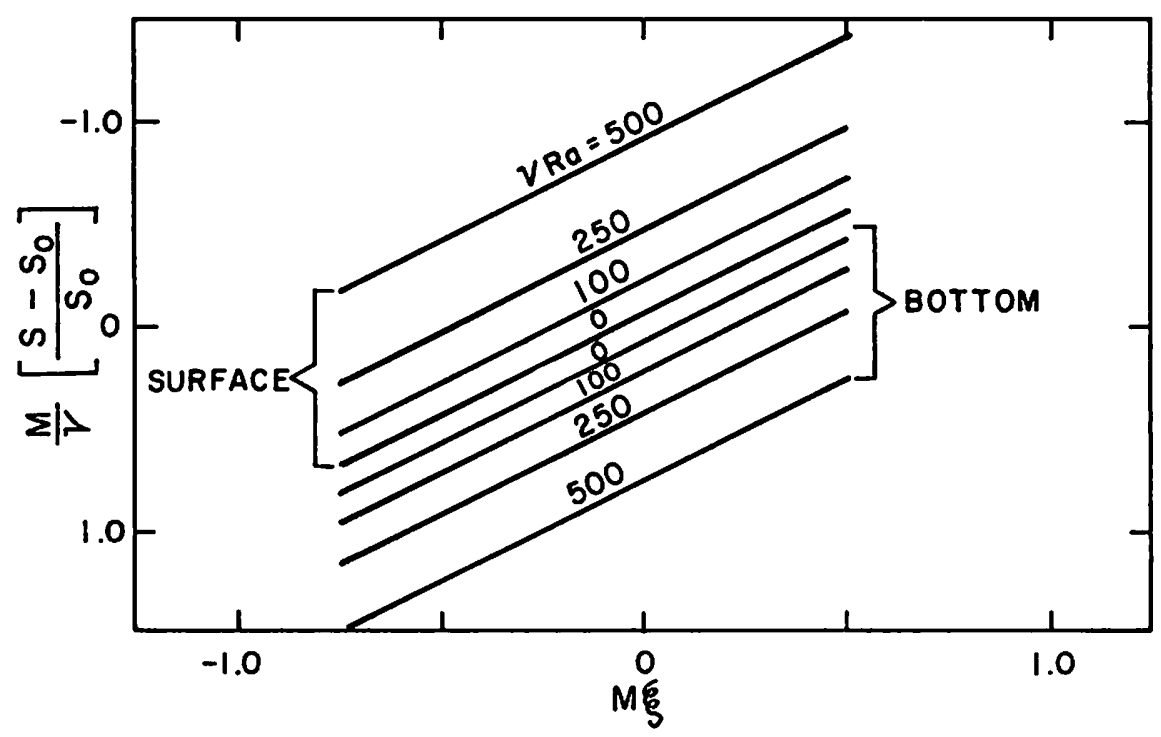

Figure 2. Salinity distributions for the central regime. 
110

Journal of Marine Research

$[23,2$

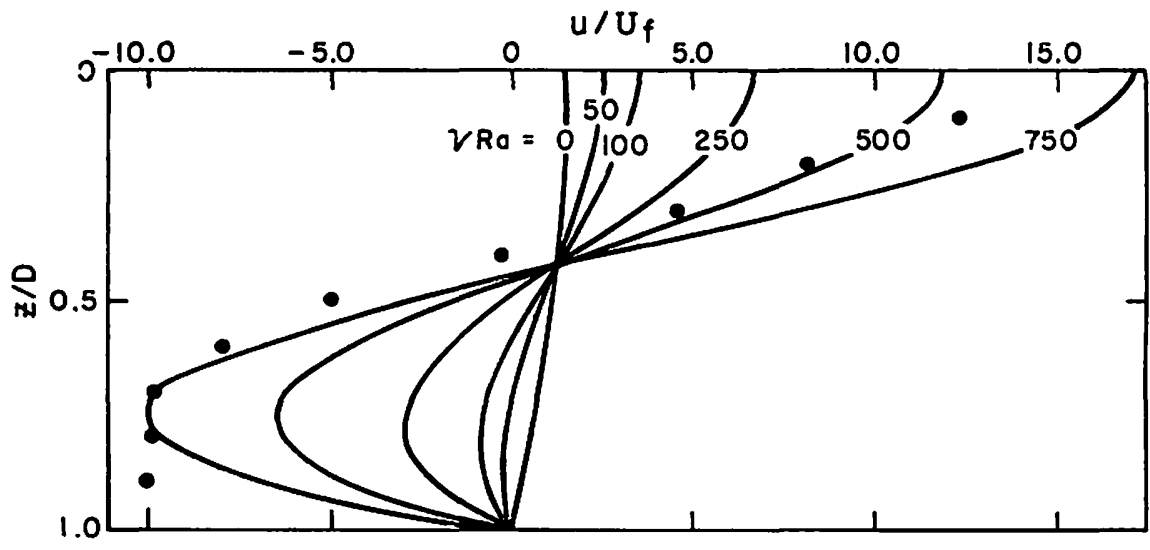

Figure 3. Horizontal velocity profile with no wind stress. Observed values (solid dots) for James River St. J17.

Equation (17) expresses the circulation as the sum of three modes: the riverdischarge mode, the wind-stress mode, and the gravitational-convection mode associated with the Rayleigh number. Only the first of these modes has a net transport of water. With no wind stress, the nature of the velocity profile depends only upon $\nu R a$ as shown in Fig. 3. When this product is zero, the velocity profile assumes the parabolic form characteristic of parallel flows having constant viscosity. As $\nu R a$ increases, the effect of the density gradient becomes apparent and the flow is bidirectional for $v R a>30$. The associated salinity profile depends upon both $\nu R a$ and $M / v$ in the manner illustrated in

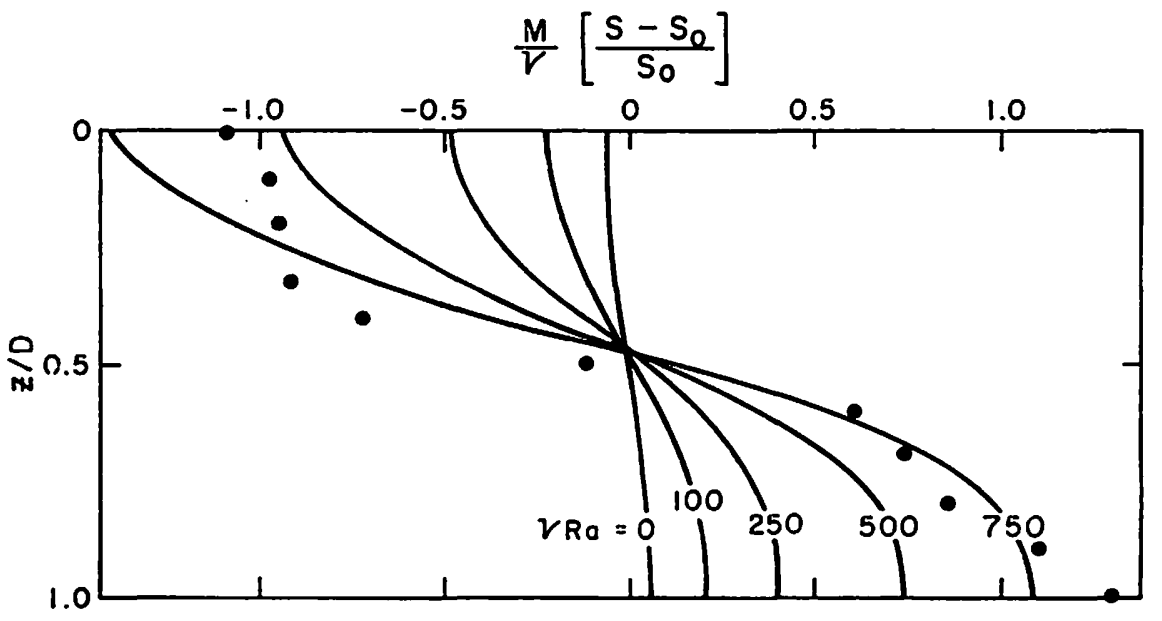

Figure 4. Salinity profiles at $\xi=0$ with no wind stress as a function of Rayleigh number and mixing parameter. Observed values (solid dots) for James River St. Jr 7 with $M / v=8$. 


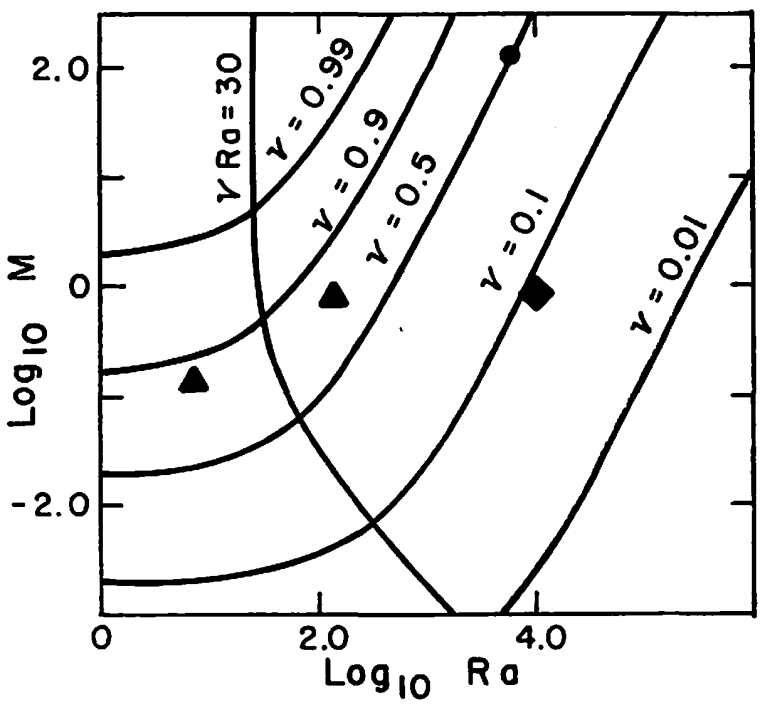

Figure 5. Solution curves for equation (18) with characteristic values for the Mersey Narrows (solid dots), James River estuary (diamonds), and Columbia River estuary (triangles).

Fig. 4. The relative stratification, like the gravitational convection, increases with $\nu R a$ but is also proportional to $\nu / M$. For large values of $\nu R a$, these profiles are asymptotic to those given by Rattray and Hansen (1962), but inclusion of the horizontal diffusion and the river discharge in the present theory

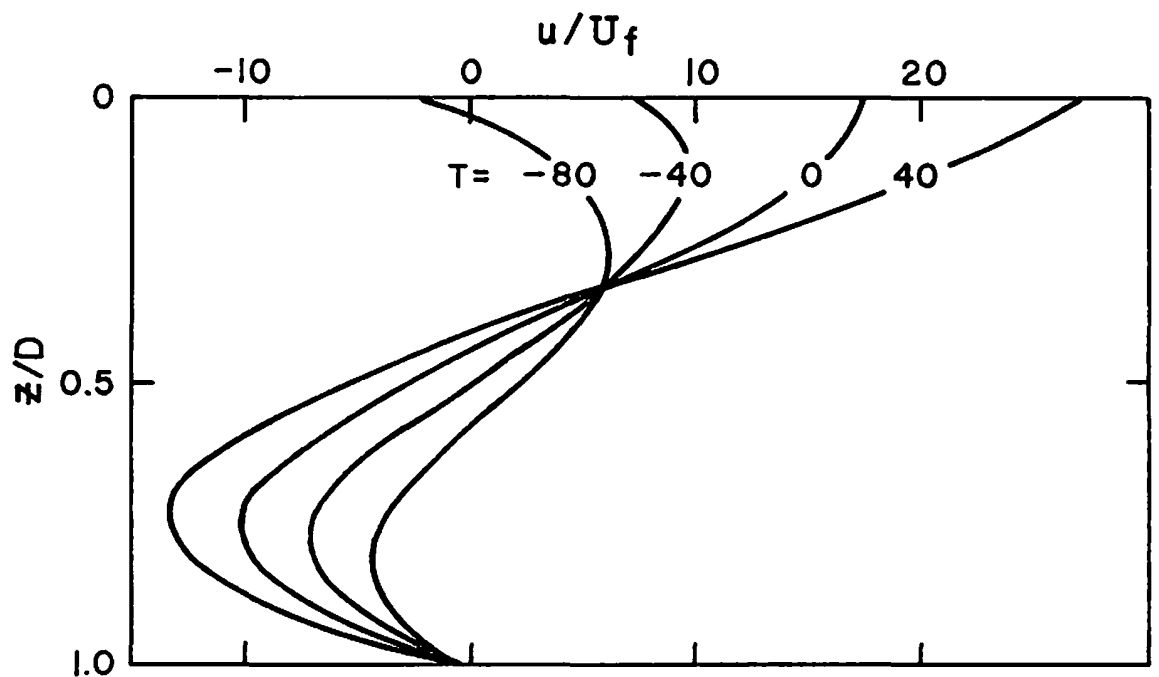

Figure 6. Modification of velocity profile by surface wind stress, with $\nu \boldsymbol{R a}=750$. 


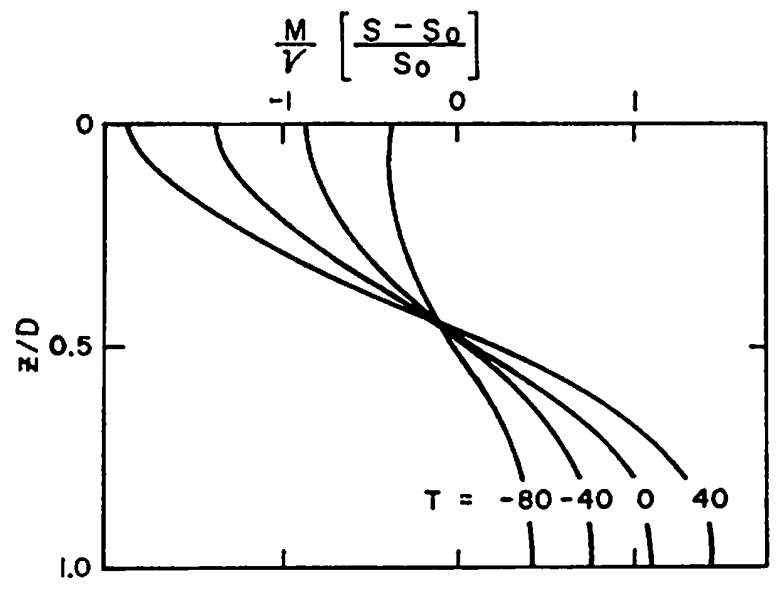

Figure 7. Modification of salinity profile at $\xi=0$ by surface wind stress, with $\nu R a=750$.

makes it possible to deduce the horizontal salinity variation from the external parameters.

The gradient parameter, $v$, is obtained from ( 14 iii) as a function of $M$, $R a$, and $T$. It is the positive root of

$1680 M(1-v)=\left(32+10 T+T^{2}\right) v+\left(76+{ }_{14} T\right) \frac{R a}{4^{8}} v^{2}+\frac{152}{3}\left(\frac{R a}{48}\right)^{2} v^{3}$.

Solutions for equation (18) in the special case of zero wind stress are shown in Fig. 5 .

Figs. 6 and 7 show the modification of the basic velocity and salinity profiles by wind stress. The significant aspects of this modification remain as described by Rattray and Hansen (1962), but quantitative inferences are more readily drawn from the present solutions. In an estuary with the characteristics of the James River, for example, $T=40$ corresponds to a wind stress of approximately 0.1 dyne $/ \mathrm{cm}^{2}-a$ rather small wind stress.

IIIb. The Central Regime; Comparison with Observations. Data given by Pritchard and Kent (1956) for the James River (St. J 17 ) are superimposed upon Figs. 3 and 4. These data demonstrate the ability of the theoretical model to represent for the most part the vertical profiles of mean velocity and salinity in estuaries, and indicate values of $\nu R a$ and $M / v$ near 750 and 8, respectively, at this station. Better agreement can be obtained by using a smaller value of $\nu R a$ and a small wind stress, but a reliable estimate of the mean wind stress is not available. Near the boundaries, good agreement cannot be expected when exchange coefficients are assumed to be independent of distance from the boundary. Direct calculation, using data given by Pritchard and Kent 
(1953, 1956), yields approximately $5 / 6$ and 9000 for $M$ and $R a$ at this station and time. However, the reference value for the horizontal diffusivity, $K_{h o}$, that enters into these determinations is probably known only within a factor of 2 or 3 . These values imply $\nu \approx 0.09$, and therefore $\nu R a \approx 800$ and $M / v \approx 9$, both of which correspond well with the values inferred from the velocity and salinity profiles.

IVa. The Inner Regime; Theory. The procedure employed by Rattray and Hansen (1962) in seeking solutions appropriate for the outer regime is applied to obtain solutions for the inner regime. Solutions are sought in the form

$$
\left.\begin{array}{l}
\psi(x, z)=R_{o} F_{i}(\gamma ; \xi) \varphi(\eta), \\
S(x, z)=S_{0} F_{i}(\nu ; \xi) \theta(\eta),
\end{array}\right\}
$$

where now $\xi=\left[R_{o} / B_{0} K_{h o}\right] x / D_{o}, \eta=F_{t}(\alpha ; \xi) z / D_{o}$; zero subscripts refer to values at $\xi=i ; F_{i}(\alpha ; \xi)$, etc., denote the functions given by Rattray and Hansen (1962), i.e.,

$$
F_{i}(\alpha ; \xi)=\left\{\begin{array}{ll}
e^{\alpha \xi} \text { if } i=0 \\
\xi^{\alpha} \text { if } i=1
\end{array}\right\} \text {, etc. }
$$

Similarity solutions are restricted in application to problems for which the external conditions are of special form. In the present case the external parameters must be expressible in the forms:

$$
\left.\begin{array}{cc}
R=R_{o} F_{i}(\gamma ; \xi), \quad D=D_{o} F_{i}(-\alpha ; \xi), & B=B_{o} F_{i}(\beta ; \xi), \\
A_{v}=A_{v o} F_{i}\left(\chi_{1} ; \xi\right), K_{v}=K_{v o} F_{i}\left(\chi_{2} ; \xi\right), & K_{h}=K_{h o} F_{i}\left(x_{3} ; \xi\right),
\end{array}\right\}
$$

subject to the similarity conditions

$$
\left.\begin{array}{l}
\beta-\gamma-4 \alpha-x_{1}=i-\nu, \\
\beta-\gamma+\alpha+x_{2}=-i, \\
\beta-\gamma-\alpha+x_{3}=i .
\end{array}\right\}
$$

The exponents $\alpha, \beta$, and $\gamma$ imply accelerations due to change in cross section and local addition of freshwater; these exponents must therefore be small to remain consistent with the neglect of acceleration terms in ( 1 ).

Substitution of (19) into (6), (7), (8), and (9), and use of (20) and (21), yield the set of ordinary differential equations and boundary conditions: 


$$
\begin{gathered}
\varphi^{\prime \prime \prime \prime}+R a\left(\nu \theta+\alpha \eta \theta^{\prime}\right)=0, \\
M \theta^{\prime \prime}+(\gamma+\nu)\left(\nu \theta+\alpha \eta \theta^{\prime}\right)+\alpha\left[\eta\left(\nu \theta+\alpha \eta \theta^{\prime}\right)\right]^{\prime}=\gamma \varphi \theta^{\prime}-\nu \varphi^{\prime} \theta, \\
\varphi(\mathrm{I})=\varphi^{\prime}(\mathrm{I})=\phi^{\prime \prime}(0)=0, \quad \varphi(0)=\mathrm{I},
\end{gathered}
$$

$$
\left.\begin{array}{c}
M \theta^{\prime}(0)-\gamma \theta(0)=0, \\
\theta^{\prime}(1)=0, \\
\nu+\int_{0}^{1}\left[\varphi^{\prime}+\alpha \eta \frac{d}{d \eta}\right] \theta d \eta=0,
\end{array}\right\}
$$

where $M$ and $R a$ are now defined with characteristic values of the parameters taken at $\xi=i$. The effect of surface wind is omitted in this section to simplify the mathematical development. If included, this effect imposes a further similarity condition upon the formulation, but its influence upon the vertical profiles of mean salinity and horizontal velocity remains as described in \$III.

The homogeneous linear system for $\theta$ given by (23) and (25) has solutions for appropriate eigenvalues of $\nu$. The nonlinear coupling with $\varphi$ makes only a single eigenvalue admissible. Solutions, applicable to estuaries with strong tidal currents, are developed by expansion in the parameter $1 / M$ :

$$
\varphi=\sum_{j=0}^{\infty} M^{-j} \varphi_{j}, \quad \theta=\sum_{j=0}^{\infty} M^{-j} \theta_{j}, \quad \nu=\sum_{j=0}^{\infty} M^{-j} \nu_{j} .
$$

Approximate solutions for $\varphi$ and $\theta$ that satisfy boundary conditions (24) and $(25 \mathrm{i})$ are:

$$
\left.\begin{array}{c}
\varphi_{0}=\frac{\mathrm{I}}{2}\left(2-3 \eta+\eta^{3}\right)-\frac{v_{0} R a}{4^{8}}\left(\eta-3 \eta^{3}+2 \eta^{4}\right), \\
\theta=1+\frac{v_{0}}{M}\left[\frac{v_{o}+\gamma}{v_{0}}\left(\eta-\frac{1}{2}\right)-\frac{1}{2}\left(v_{0}+\gamma+\alpha\right)\left(\eta^{2}-\frac{1}{3}\right)-\right. \\
-\int_{0}^{\eta} \varphi_{0} d \eta^{\prime}+\int_{0}^{1} \int_{0}^{\eta} \varphi_{0} d \eta^{\prime} d \eta .
\end{array}\right\}
$$

Derivation of a characteristic equation for $v_{o}$ from (25 ii) yields approximate values of $\boldsymbol{\nu}$ that are applicable only to estuaries having negligible stratification or gravitational convection. A more general result is obtained by using (25 iii), which leads to the characteristic equation, 


$$
\begin{gathered}
1680 M\left(1-v_{0}\right)=210(1+4 \alpha) \gamma+ \\
+\left[144+\left(252 \frac{R a}{48}-112-560 \alpha\right) \gamma-560(\alpha-1) \alpha\right] v_{0}+ \\
+\left[(188-112 \gamma) \frac{R a}{48}-112-560 \alpha\right] \nu_{o}^{2}+ \\
+\left[\frac{152}{3}\left(\frac{R a}{48}\right)^{2}-112 \frac{R a}{48}\right] v_{0}^{3}
\end{gathered}
$$

The vertical salinity profiles given by (27) are influenced to some extent by longitudinal variation in cross section and freshwater discharge, but for large values of $v R a$, these profiles closely resemble those in Fig. 4. The difference in behavior is primarily near the boundaries, where the normal derivative may not vanish because of freshwater injection at the surface and because approximate values of $v$ obtained from (25 iii) do not generally satisfy (25 ii) exactly.

The horizontal velocity profile implied by (26) is the same as that shown in Fig. 3; however, whereas the special form of similarity required in $\$$ III allowed only strictly parallel, unaccelerated flow, solutions in the form of (19) yield the vertical velocity given by

$$
W=\frac{K_{v o}}{M D_{0}} F_{i}(\gamma-i ; \xi)\left[\gamma \varphi+\alpha \eta \varphi^{\prime}\right] .
$$

The first term in the bracket is a contribution to the vertical velocity associated with local addition of river water, and the second term is a contribution associated with bottom slope. The nature of each of these contributions is shown in Figs. 8 and 9.

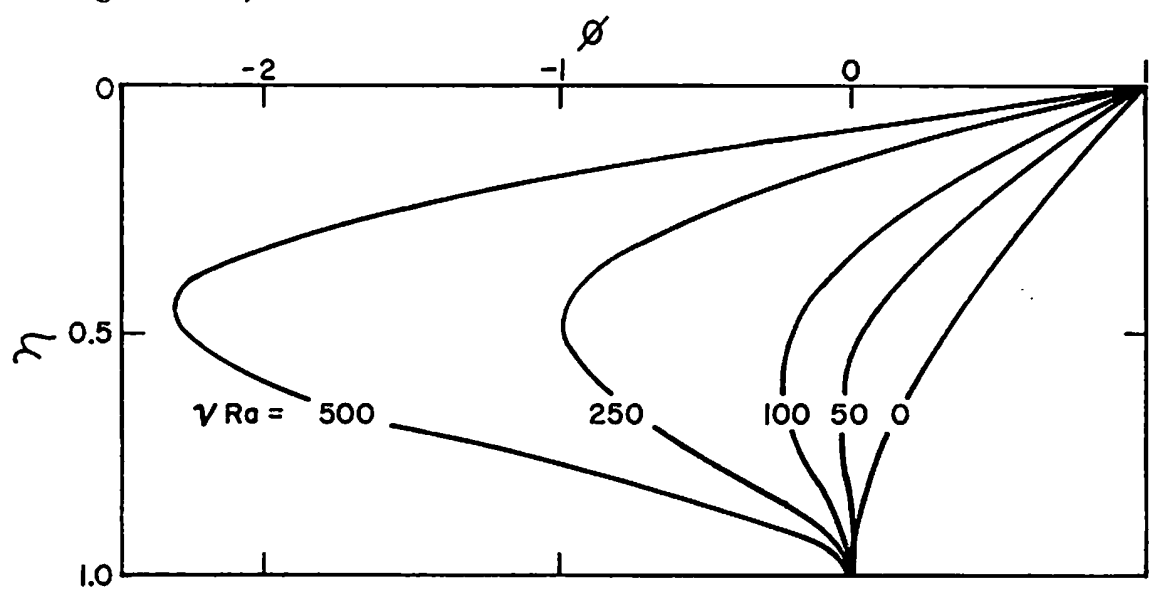

Figure 8. Freshwater addition contribution to vertical velocity. 


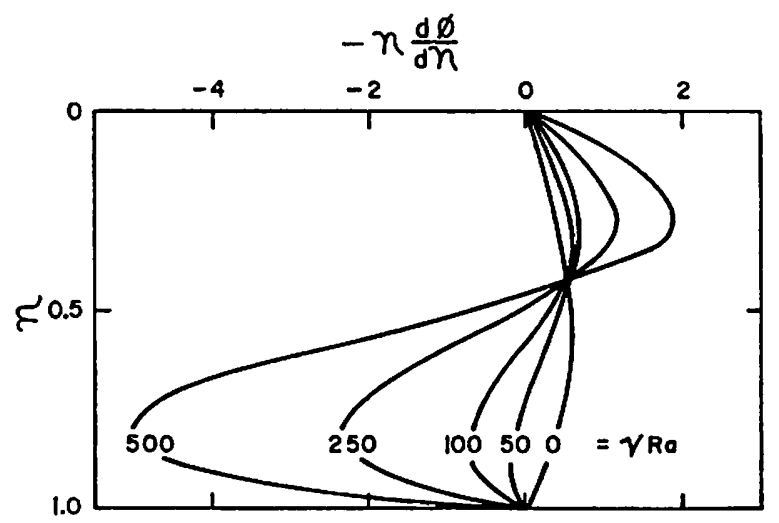

Figure 9. Bottom slope contribution to vertical velocity.

IVb. The Inner Regime; Comparison with Observations. A variety of estuarine situations can be described by specifying the function $F_{\mathfrak{l}}$ and the exponents $\alpha, \beta$, and $\gamma$. A simple example, in which the estuary is that of a tidal river having uniform width and depth and in which the salinity intrusion is controlled by dynamic conditions rather than by the physical length of the inlet, is obtained by taking $i=\alpha=\beta=\gamma=0$. The similarity conditions then require that $K_{h}$ and $K_{v}$ be independent of $x$ and that $A_{v}$ be proportional to the

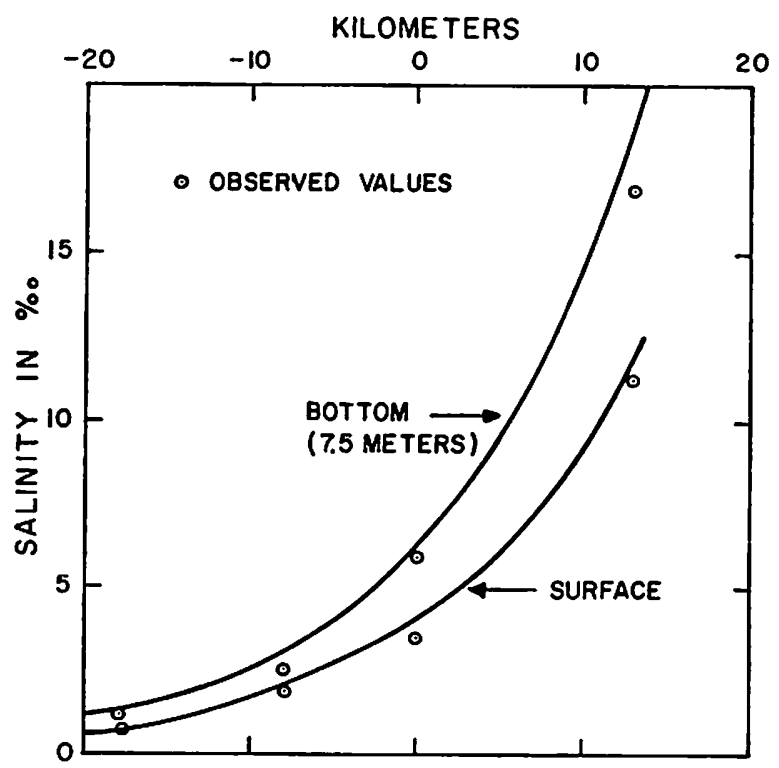

Figure 10. Longitudinal variation in surface and bottom salinity in inner part of the James River estuary. 


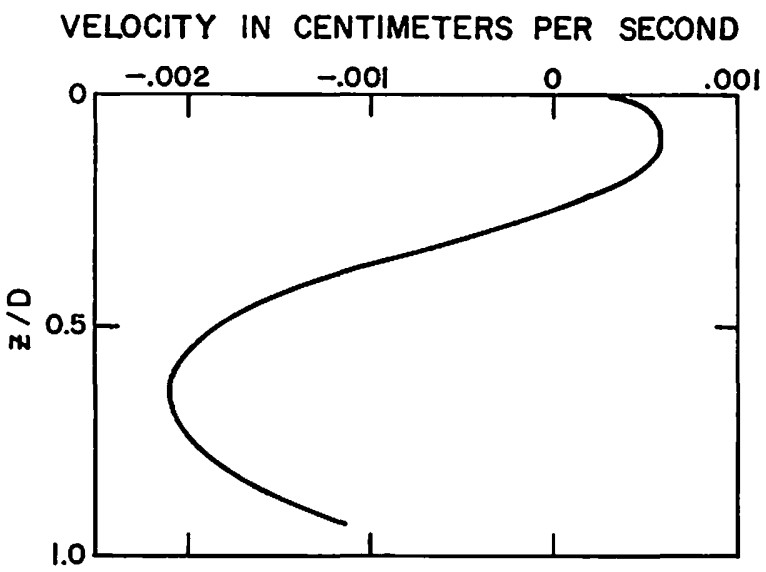

Figure I1. Typical profile of vertical velocity at St. J24, James River estuary.

mean salinity and its gradient. The inner part of the James River estuary can be interpreted in terms of this simple model. Data from Pritchard and Kent (1953, 1956) indicate values of $M$ and $R a$ near $5 / 6$ and 3000 , respectively, if the reference section is placed at St. J 24. The appropriate root of equation (28), with these values, is $\nu \approx 0.16$. Fig. I shows the theoretical longitudinal salinity distribution in comparison with that observed at a time of comparable river flow. The observed data are not mean values over the tidal cycle, but the general agreement is good.

Although the vertical velocities calculated by Pritchard and Kent (1953) for the James River estuary are in general of a type that cannot be portrayed exactly by the similarity solutions, they closely resemble those in Fig. 8 except near the surface. However, their calculated profiles at St. J24 (sample in Fig. II) consistently show features that have no apparent explanation except for the bathymetric effect shown in Fig. 9.

V. Discussion. Many investigators, motivated by Knudsen's (1900) early work or perhaps by misinterpretation of the significance of two-layer models such as that of Defant (1961:517), continue to draw inferences concerning the mean velocity from the salinity distribution in estuaries. Results from the present work indicate that such inferences can be misleading when turbulent diffusion is an important mechanism for horizontal salt flux. Except for windstress effects, the products $\nu R a$ and $M / \nu$ specify the vertical profiles of salinity and mean velocity in the central region, and, for large values of $\nu R a$, they dominate those in the inner region. As $M / v \rightarrow \infty$, the salinity distributions are asymptotic to the one-dimensional distribution; this has frequently been considered to be indicative of a net seaward flow at all depths. Figs. 3 and 4 indicate that a sectionally homogeneous salinity distribution does not imply a uni- 
directional mean flow except to the extent that $M$ and $R a$ are inversely correlated. Some correlation is to be expected, but the strong influence of the total depth on the Rayleigh number indicates that deep estuaries in particular may have well-developed gravitational convection even though tidal mixing nearly destroys the vertical salinity gradient. Hansen (1964) has pointed out in this regard that, although the Narrows of the Mersey Estuary in England is usually much less stratified than the James River, the greater depth in the Narrows permits greater development of gravitational convection. A further example can be made of the Columbia River estuary, for which observations cited by Lockett (1963) indicate that gravitational convection is but slightly developed even though there is marked salinity stratification.

The gradient parameter, $v$, is the fraction of the salt advected seaward with the river discharge that is balanced by upstream salt flux associated with fluctuating motions. It may also be interpreted as the ratio by which the upstream advection of salt (and the consequent extension of the salinity intrusion) reduces the horizontal salinity gradient from the gradient that would be required to balance seaward advection of salt by turbulent diffusion with a fixed value of $K_{h o}$. It is thus seen that, although the gravitational convection mode of circulation does not give a net flux of water, it does, when coupled with vertical variation of salinity, give a net upstream salt flux. As found by Pritchard (1954b), this flux may nearly balance the salt advected seaward by the riverdischarge mode. Bowden (1963) has shown that this advective salt flux may be largely responsible for the very large apparent diffusion coefficients reported by Hughes (1958) for the Mersey Narrows. These apparent diffusion coefficients, which combine the upstream salt flux by this advective mechanism of the mean flow with that due to fluctuating motions, are not applicable in estimating the movement and dispersal of concentrations of scales other than that of the salinity intrusion. Their use is liable to be particularly misleading when applied to concentrations of substances that seek a preferred depth by virtue of density or motility. A sectionally homogeneous salinity distribution does not imply the absence of gravitational convection. While it does imply that gravitational convection cannot contribute to the salt balance, this statement applies to other concentrations only if their distribution is also sectionally homogeneous.

An important facet of the salinity-velocity interaction is shown by the dependence of $\nu$ upon $M$ and $R a$. It is apparent in Fig. 5 that $\nu$ increases and decreases respectively as $M$ and $R a$ increase, forming a negative feedback loop that tends to stabilize $M / v$ and $v R a$-hence, to stabilize the vertical profiles of salinity and velocity - against variations as great as those in $M$ and $R a$. Further, from (10) or (19) it is seen that

$$
\frac{1}{S_{o}} \frac{\partial S}{\partial x} \propto v \frac{U_{f}}{K_{h o}}
$$


indicating that, if the exchange coefficients are not strongly dependent upon freshwater runoff, then the explicit dependence of the horizontal salinity gradient upon $R$ tends to be offset by the dependence of $\nu$ upon $R$. The physical explanation of this control mechanism lies in the tendency for both horizontal and vertical salinity gradients to increase with river discharge. Such increases in turn cause greater upstream salt advection, thus reducing somewhat the horizontal salinity gradient required to maintain the salt balance at the new discharge level. Cameron and Pritchard (1963) have described a tendency for estuaries to be buffered against changes in salinity as great as those in river discharge. This tendency is an estuarine analog of Le Châtelier's principle, in which changes in the regime resulting from variations in the parameters comprising $M$ and $R a$ are opposed by adjustment of the advective salt flux mechanism. When this mechanism plays a large role in maintaining the salt balance, the required adjustment may be effected by relatively small changes in the horizontal and vertical salinity gradients.

A priori knowledge of the exchange coefficients required to determine the distributions of mean velocity and salinity from the theory are of course not always available. However, from salinity and velocity observations, the theoretical results can be used to obtain rapid estimates of effective values of these coefficients. Observed profiles of mean velocity and salinity define values of $\nu R a$ and $M / v$, from which $\nu$ may be evaluated by means of (18) or (28). Knowledge of the horizontal salinity gradient then permits estimation of all three exchange coefficients involved in the theory. Table I and Fig. 5 show values of these parameters for some representative estuaries. The values deduced for the James River and the Mersey Narrows agree well with the results of more detailed analyses by Pritchard (1945 b, 1956) and Bowden (1963). Detailed studies have not been made of the dynamics of either the Columbia River or the Strait of Juan de Fuca off Washington. Note that the horizontal diffusivity obtained in this manner for the Columbia River estuary, unlike the apparent diffusion coefficients found by Hughes (1958) for the Mersey Narrows, is independent of the river discharge.

TABLE I. VAlues of Parameters Inferred from Distributions of Velocity and Salinity in Particular Estuarine Regions.

$\begin{array}{rrrrrrr}\text { Columbia River } & \nu R a & M / \nu & \nu & \begin{array}{c}A_{v} \\ \mathrm{~cm}^{2} / \mathrm{sec}\end{array} & \begin{array}{c}K_{v} \\ \mathrm{~cm}^{2} / \mathrm{sec}\end{array} & \begin{array}{c}K_{h} \\ 10^{6} \mathrm{~cm}^{2} / \mathrm{sec}\end{array} \\ \text { High stage ........ } & <30 & 0.1-0.2 & 0.9 & >25 & 15-30 & 51 \\ \text { Low stage........ } & 30-250 & 0.5-2 & 0.8 & 5-50 & 5-20 & 46 \\ \text { James River ........ } & 750 & 8 & 0.1 & 2.5 & 1 & 0.24 \\ \text { Mersey Narrows .... } & 3000 & 270 & 0.5 & 30 & 12 & 1 \\ \text { Juan de Fuca Strait*. } & 9000 & 450 & - & 75 & 15 & -\end{array}$

* Based primarily upon data by Herlinveaux (1954) and Waldichuck (1957). 
Some practical limitations are encountered in this application of the theory. In estuaries such as the Columbia River, where the influence of the salinity gradient on the velocity profile is not large compared with the influences of bathymetry, bed roughness, and error of measurement, it is difficult to obtain accurate estimates of $\nu R a$ and $M / v$. This difficulty, although it leads to uncertainty in determination of the vertical exchange coefficients, does not greatly influence the horizontal diffusivity, which dominates the upstream salt flux in this situation. On the other hand, (18) or (28) may be considered as an equation for $v$ in terms of $\nu R a$ and $M / v$; this is equivalent to determining the turbulent salt flux as the difference between the landward and seaward advective fluxes. When this difference is a small fraction of the advective fluxes, it is also highly sensitive to slight differences in profile shape, errors in estimating $\nu R a$ and $M / \nu$, and departure of actual cross sections from the rectangular form assumed in the theory. In this instance, proper estimates of the vertical exchange coefficients are obtained, but the estimated horizontal diffusivity tends in general to be low or even negative. These considerations restrict application of the models to values of $\nu R a$ greater than 30 for estimation of the vertical exchange coefficients, and perhaps to $v$ greater than 0.1 for estimation of the horizontal diffusivity. These limits define a region of applicability in the $M-R a$ plane (cf. Fig. 5). A lower limit of detectable stratification may be expected to set an upper bound on usable values of $M / v$, but this situation has not been encountered.

VI. Conclusion. Similarity solutions have been obtained for the coupled system of partial differential equations describing the circulation and salt balance for a wide range of estuarine conditions. These solutions give relations among the external parameters that determine the nature of particular estuarine regimes and provide new insight into the interaction of the salinity and velocity fields as a control mechanism in estuarine dynamics. The circulation is conveniently separated into three modes: the freshwater discharge or forced mode, the gravitational convection or induced mode, and a wind-stress mode. Only the first of these modes gives a net transport of water, but all may contribute to the longitudinal salt balance.

While gravitational convection results from the horizontal salinity gradient and acts to form a vertical salinity gradient, the velocity distribution in partially mixed estuaries cannot be inferred from the salinity structure alone. The mean flow and the salinity stratification are strongly coupled, but they have different dependencies on the two dimensionless quantities that characterize the circulation. Deeper estuaries in particular are likely to have a reversal of mean velocity at depth, even with only a slight salinity stratification. Disregard for the effect of advective processes in maintaining the salinity distribution in estuaries will generally yield misleading estimates of the movement and mixing of other concentrations in an estuary. 


\section{REFERENCES}

Aввот,, M. R.

1960. Salinity effects in estuaries. J. Mar. Res, I8 (2): ror-I I I.

AGNEW, RODERICK

1961. Estuarine currents and tidal streams. Proc. $7^{\text {th }}$ Conf. coast. Engng., The Hague, 1960, 2: (Ch. 28) 510-535.

Arons, A. B., and Henry Stommel

1951. A mixing-length theory of tidal flushing. Trans. Amer. geophys. Un., 32 (3): $419-421$.

BOWDEN, K. F.

1963. The mixing processes in a tidal estuary. Int. J. Air Wat. Poll., 7 (4-5): 343356.

Cameron, W. M., and D. W. Pritchard

1963. Estuaries. In The Sea. M. N. Hill, Ed. Interscience, New York. 2 (Ch. 15): 306324. $554 \mathrm{pp}$.

Defant, Albert

1961. Physical oceanography. Pergamon Press, New York. 729 Pp.

HANSEN, D. V.

1964. Salt balance and circulation in partially-mixed estuaries. Proc. Conf, on Estuaries, Jekyll Island, 1964 .

HeRlinveaux, R. H.

1954. Tidal currents in Juan de Fuca Strait. J. Fish. Res. Bd. Canada, II (6): 799815.

Hughes, Peter

1958. Tidal mixing in the Narrows of the Mersey Estuary. Geophys. J., I (4): 27I283.

IPPEN, A. T., and D. R. F. HARLEMAN

1961. One-dimensional analysis of salinity intrusion in estuaries. Tech. Bull. Comm. tid. Hydraul., U.S. Army, 5; 52 pp.

KENT, R. E.

1958. Turbulent diffusion in a sectionally homogeneous estuary. Tech. Rep. Chesapeake Bay Inst., I6, Ref. 58-1; 86 pp.

KNUDSEN, MaRTin

1900. Ein hydrographischer Lehrsatz. Ann. Hydrog. marit. Meteorol., 28: 316.

LOCKETT, J. B.

1963. Phenomena affecting improvement of the lower Columbia estuary and entrance. Proc. 8th Conf. coast. Engng., Mexico City, 1962, Ch. 40: 695-755. 810 pp.

O'Brien, M. P.

1952. Salinity currents in estuaries. Trans. Amer. geophys. Un., $33(4): 520-522$.

Preddy, W. S.

1954. The mixing and movement of water in the estuary of the Thames. J. Mar. biol. Ass. U. K., 33 (3): 645-662.

Pritchard, D. W.

1954a. A study of flushing in the Delaware model. Tech. Rep. Chesapeake Bay Inst., 7, Ref. 54-4; 143 PP.

1954 b. A study of the salt balance of a coastal plain estuary. J. Mar. Res., 13 ( 1 ): $133^{-144}$. 1956. The dynamic structure of a coastal plain estuary. J. Mar. Res., $15(1): 33-42$. 
Pritchard, D. W., and R. E. Kent

1953. The reduction and analysis of data from the James River Operation Oyster Spat. Tech. Rep. Chesapeake Bay Inst., 6, Ref. 53-12; 92 pp.

1956. A method for determining mean longitudinal velocities in a coastal plain estuary. J. Mar. Res., 15 (1): 8I-91.

Rattray, Maurice, JR., and D. V. Hansen

1962. A similarity solution for circulation in an estuary. J. Mar. Res., 20 (2): $121-133$. Waldichuck, Mrchael

1957. Physical oceanography of the Strait of Georgia, British Columbia. J. Fish. Res. Bd. Canada, $14(3): 321-486$. 